\title{
Saddles for expansive flows with the pseudo orbits tracing property
}

\author{
by JERzY OMBACH (Kraków)
}

\begin{abstract}
Let $F$ be an expansive flow with the pseudo orbits tracing property on a compact metric space $X$. Suppose $X$ is connected, locally connected and contains at least two distinct orbits. Then any point is a saddle.

1. Introduction. This paper follows paper [6] where we have studied expansive flows having the pseudo orbits tracing property on a compact metric space. Such flows were identified and studied by R. Thomas in [9]$[12]$ and we refer the reader to those papers for basic information. In [6] we have characterized three possible types of behavior of orbits near a given point as well as near a basic set pointing out that they are similar to the behavior of orbits near hyperbolic points or sets in the differential case. We have classified points as sinks, sources or saddles. The aim of the present paper is to prove the following.
\end{abstract}

1.1. TheOrem. Let $X$ be a compact metric space, connected, and locally connected, and $F$ an expansive flow on $X$ having the pseudo orbits tracing property. If $X$ contains two distinct orbits then every point is a saddle.

The corresponding result for expansive homeomorphisms with canonical coordinates is due to Reddy and Robertson (see [8]). Using the suspension flow procedure one can derive that result from Theorem 1.1 because Thomas [9] proved that the suspension flow of an expansive homeomorphism with the pseudo orbits tracing property is expansive and has the pseudo orbits tracing property and Reddy [7] showed that the canonical coordinates plus expansiveness imply the pseudo orbits tracing property (see also [4] and [5]).

We also note that if a compact space $X$ is a single orbit then $X$ must be a point or be homeomorphic to a circle. In either case any flow on $X$ is

1991 Mathematics Subject Classification: 58F15.

Supported by Polish Scientific Grant RP.1.10. 
expansive and has the pseudo orbits tracing property. There are no saddles since every point is a sink and a source.

The proof of Theorem 1.1 is carried out in Section 3 and its general idea comes from [8]. Yet, the details are different. We have to use the concept of a local cross-section. Moreover, we cannot apply a theorem of Gottschelk and Hedlund as in [8]. We take advantage of a result of [6] instead. Section 2 contains basic definitions and the main results of [6] needed in Section 3. Section 4 is independent and is devoted to the study of the coordinates that have been identified in Section 2 and found extremely useful in Section 3.

2. Definitions. In this section we recall basic definitions and properties needed in Section 3. We assume in this paper that $(X, d)$ is a compact metric space and $F$ is a flow on $X$, i.e. $F: X \times \mathbb{R} \ni(x, t) \rightarrow F(x, t)=x t \in X$ is continuous, $x 0=x, x(t+s)=(x t) s$. We also assume that $F$ does not have fixed points (this is not a real restriction if we study expansive flows, see $[2])$.

Define

$$
\begin{gathered}
B(x, \varepsilon)=\{y: d(x, y) \leq \varepsilon\}, \quad B_{\varepsilon}=\{(x, y): d(x, y) \leq \varepsilon\}, \\
S \cdot J=\{y: y=x t, x \in S, t \in J\} \quad \text { for } S \subset X, J \subset \mathbb{R},
\end{gathered}
$$

$\operatorname{Rep}(J)=\{h: J \rightarrow J: h$ is an increasing homeomorphism, $h(0)=0\}$, where $J$ is an interval, $0 \in J$,

$$
\begin{gathered}
W_{\varepsilon}^{s}(x)=\{y: \text { there exists } h \in \operatorname{Rep}[0, \infty) \text { such that } \\
\quad d(x t, y h(t)) \leq \varepsilon \text { for } t \geq 0\}, \\
\begin{aligned}
W_{\varepsilon}^{u}(x)=\{y: \text { there exists } h \in \operatorname{Rep}(-\infty, 0] \text { such that } \\
d(x t, y h(t)) \leq \varepsilon \text { for } t \leq 0\}, \\
D_{\beta, r}=\{(x, y): d(x t, y) \leq \beta, d(x, y s) \leq \beta \text { with some }|t|,|s| \leq r\} .
\end{aligned}
\end{gathered}
$$

We say that the flow $F$ is expansive if for every $r>0$ there exists $e>0$ such that for any $x, y \in X$ and $h \in \operatorname{Rep}(\mathbb{R})$ the inequality $d(x t, y h(t)) \leq e$ for all $t \in \mathbb{R}$ implies $y=x t$ with some $|t| \leq r$.

$\mathrm{A}(\delta, 1)$-pseudo orbit is a pair of doubly infinite sequences $\left\{x_{n}\right\}_{n \in \mathbb{Z}} \subset X$, $\left\{t_{n}\right\}_{n \in \mathbb{Z}} \subset \mathbb{R}$ such that for any $n \in \mathbb{Z}$

$$
d\left(x_{n} t_{n}, x_{n+1}\right) \leq \delta \quad \text { and } \quad t_{n} \geq 1 .
$$

For a given $(\delta, 1)$-pseudo orbit we denote by $x_{0} * t$ the point on this pseudo orbit $t$ units from $x_{0}$. More precisely:

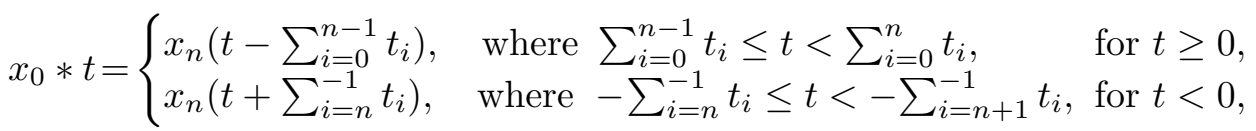
where $\sum_{m}^{n}()=0$ if $n<m$. 
A $(\delta, 1)$-pseudo orbit is $\varepsilon$-traced if there exist $x \in X$ and $h \in \operatorname{Rep}(\mathbb{R})$ such that for any $t \in \mathbb{R}$

$$
d\left(x h(t), x_{0} * t\right) \leq \varepsilon .
$$

We say that $F$ has the pseudo orbits tracing property if for every $\varepsilon>0$ there exists $\delta>0$ such that any $(\delta, 1)$-pseudo orbit is $\varepsilon$-traced.

For the rest of this section we assume that the flow $F$ is expansive and has the pseudo orbits tracing property. We quote certain properties of $F$ from $[6]$.

2.1. Theorem (Proposition 2.9 in [6]). For any $r>0$ there exists $\varepsilon_{r}>0$ with the property that for every $0<\varepsilon \leq \varepsilon_{r}$ there exist $\delta>0$ and a map $[,]_{r, \varepsilon}: B_{\delta} \rightarrow 2^{X}$ such that:

(a) for any $(x, y) \in B_{\delta}$

$$
[x, y]_{r, \varepsilon}=W_{\varepsilon}^{s}(x) \cap W_{\varepsilon}^{u}(y) \neq \emptyset,
$$

(b) for any $(x, y) \in B_{\delta}$ and $z, w \in[x, y]_{r, \varepsilon}$ there exists $|t| \leq r$ with $w=z t$,

(c) the following continuity condition holds: for every $\beta>0$ there exists $\alpha>0$ such that for any $(x, y),\left(x^{\prime}, y^{\prime}\right) \in B_{\delta}$ satisfying $d\left(x, x^{\prime}\right) \leq \alpha, d\left(y, y^{\prime}\right) \leq$ $\alpha$ we have

$$
[x, y]_{r, \varepsilon} \times\left[x^{\prime}, y^{\prime}\right]_{r, \varepsilon} \subset D_{\beta, r} .
$$

2.2. Remark. Conditions (a) and (b) in Theorem 2.1 will serve as a definition of coordinates in Section 4. The proof of Proposition 2.9 in [6] shows that, in fact, the continuity condition (c) is a consequence of (a) and (b).

2.3. ThEOREM (Theorem 3.7 in [6]). For every $r>0$ there exists $\varepsilon_{r}>0$ such that for any fixed $\varepsilon$ with $0<\varepsilon \leq \varepsilon_{r}$ and points $x \in X$ and $y \in \omega(x)$ $(\omega(x)$ is the $\omega$-limit set of $x)$ the following conditions are equivalent:

(a) $\operatorname{int} W_{\varepsilon}^{s}(x) \neq \emptyset$,

(b) $x \in \operatorname{int} W_{\varepsilon}^{s}(x)$,

(c) there exists a neighborhood $H$ of $x$ such that $W_{\varepsilon}^{u}(x) \cap H \subset x[-r, r]$,

(d) $y$ is periodic, $y \in \operatorname{int} W_{\varepsilon}^{s}(x)$,

(e) $\omega(x)$ is a periodic orbit and $z \in \operatorname{int} W_{\varepsilon}^{s}(z)$ for any $z \in \omega(x)$.

Moreover, if any of the above conditions holds for some $0<\varepsilon \leq \varepsilon_{r}$ then all of them hold for any $0<\varepsilon \leq \varepsilon_{r}$.

A point $x$ is said to be a sink if $x \in \operatorname{int} W_{\varepsilon}^{s}(x)$ with small $\varepsilon>0$, a source if it is a sink for the reverse flow $F^{\prime}(x, t)=x(-t)$ and a saddle otherwise. Assume that $x$ is a flow non-isolated point, i.e. for any $r>0$ and any neighborhood $U$ of $x, U \backslash x[-r, r] \neq \emptyset$ (in fact, we may assume that $r>0$ is fixed and $r \leq T_{0}$, where $T_{0}>0$ is the infimum of the periods of periodic 
orbits; cf. Lemma 2 in [2] or Lemma 2.3 in [6]). Such an $x$ cannot be a sink and a source at the same time; it is a saddle iff any neighborhood of $x$ contains points from $W_{\varepsilon}^{s}(x) \backslash x[-r, r]$ and from $W_{\varepsilon}^{u}(x) \backslash x[-r, r]$.

2.4. Theorem (cf. Lemma 4.1 in [1], Propositions 2.12 and 2.13 in [6]).

(a) $\operatorname{cl}$ Per $=\operatorname{cl} \alpha=\operatorname{cl} \omega=\Omega=\mathrm{CR}$, where Per is the set of periodic points, $\alpha$ the set of $\alpha$-limit points, $\omega$ the set of $\omega$-limit points, $\Omega$ the set of non-wandering points and $\mathrm{CR}$ the set of chain recurrent points.

(b) $\Omega=\Omega_{1} \cup \ldots \cup \Omega_{r}$, where the $\Omega_{i}$ are closed, invariant and pairwise disjoint.

(c) For each $i=1, \ldots, r, \Omega_{i}$ is topologically transitive and any two points $x, y \in \Omega_{i}$ are chain equivalent.

The sets $\Omega_{i}$ in Theorem 2.4 are said to be basic sets.

2.5. Theorem (cf. Theorem 3.12 in [6]). Let $K$ be a basic set. There exists $\varepsilon_{1}>0$ such that the following conditions are equivalent:

(a) int $W_{\varepsilon}^{s}(K) \neq \emptyset$ for some $\varepsilon$ with $0<\varepsilon \leq \varepsilon_{1}$,

(b) $W^{s}(K)$ is open,

(c) $W^{u}(K)=K$,

(d) int $W_{\varepsilon}^{s}(K) \neq \emptyset$ for each $\varepsilon$ with $0<\varepsilon \leq \varepsilon_{1}$.

Here $W_{\varepsilon}^{s}(K)=\{x \in X: d(x t, K) \leq \varepsilon$ for $t \geq 0\}, W^{s}(K)=\{x \in X:$ $x t \rightarrow K$ as $t \rightarrow \infty\}$ and $W^{u}(K)=\{x \in X: x t \rightarrow K$ as $t \rightarrow-\infty\}$.

2.6. Proposition (cf. Proposition 3.13 in [6]). If a periodic point $y$ is a sink/source then its orbit is a basic set.

3. Proof of Theorem 1.1. We assume in this section that $F$ is an expansive flow on a compact metric space $X$ having the pseudo orbits tracing property.

3.1. Lemma. For every $\varepsilon>0$ there exist $\varepsilon^{\prime}>0$ and $\tau>0$ such that if $y \in W_{\varepsilon^{\prime}}^{s}(x)$ (resp. $\left.y \in W_{\varepsilon^{\prime}}^{u}(x)\right)$, then $y[-\tau, \tau] \subset W_{\varepsilon}^{s}(x)$ (resp. $y[-\tau, \tau] \subset$ $\left.W_{\varepsilon}^{u}(x)\right)$.

Proof. It is enough to prove the lemma for the case of $W_{\varepsilon}^{s}(x)$. Let $\varepsilon>0$. Continuity of the flow provides $\tau_{1}>0$ such that

$$
d(z t, z s) \leq \varepsilon / 3 \quad \text { for all } z \in X,|t|,|s| \leq \tau_{1} .
$$

We assume that $\tau_{1}<1$ and put $\tau=\tau_{1} / 6$. Lemma 1.2 in [11] provides $\varepsilon^{\prime}>0$ with $\varepsilon^{\prime} \leq \varepsilon / 3$ such that if for some $z \in X$ and $h \in \operatorname{Rep}[0, \infty)$, $d(z h(t), x t) \leq \varepsilon^{\prime}$ for all $t \geq 0$, then

$$
|h(t)-t| \leq \tau \quad \text { for } 0 \leq t \leq 1 .
$$


Let $y \in W_{\varepsilon^{\prime}}^{s}(x)$, i.e.

$$
d(x t, y h(t)) \leq \varepsilon^{\prime} \quad \text { for all } t \geq 0
$$

with some $h \in \operatorname{Rep}[0, \infty)$, and let $z=y s$ with $|s| \leq \tau$. We want to show that $z \in W_{\varepsilon}^{s}(x)$.

Assume first that $s>0$. There is $t_{0}>0$ such that $h\left(t_{0}\right)=s$. We claim that $t_{0} \leq 2 \tau$. We have $h\left(t_{0}\right)=s \leq \tau<1-\tau$ and, by (2) with $t=1$, $h\left(t_{0}\right) \leq h(1)$, hence $t_{0} \leq 1$. Again (2) with $t=t_{0}$ implies $t_{0} \leq h\left(t_{0}\right)+\tau=$ $s+\tau \leq 2 \tau$, which proves the claim. Define $g \in \operatorname{Rep}[0, \infty)$ by

$$
g(t)= \begin{cases}\frac{h\left(2 t_{0}\right)-s}{2 t_{0}} t & \text { for } 0 \leq t \leq 2 t_{0} \\ h(t)-s & \text { for } 2 t_{0} \leq t\end{cases}
$$

For $0 \leq t \leq 2 t_{0}$ we have

$$
d(z g(t), x t) \leq d(z g(t), z(h(t)-s))+d(y h(t), x t) \leq \varepsilon / 3+\varepsilon^{\prime}<\varepsilon,
$$

where the second term is $\leq \varepsilon^{\prime}$ by (3) and the first one is $\leq \varepsilon / 3$ by (1) since from (2) we have $g(t) \leq h\left(2 t_{0}\right) \leq 2 t_{0}+\tau \leq 5 \tau \leq \tau_{1}$ and $|h(t)-s| \leq$ $h(t)+s \leq h\left(2 t_{0}\right)+s \leq 5 \tau+\tau=\tau_{1}$. Note that $2 t_{0} \leq 4 \tau<1$. For $2 t_{0} \leq t$ we have, by $(3), d(z g(t), x t)=d(y h(t), x t) \leq \varepsilon^{\prime}<\varepsilon$. Hence $z \in W_{\varepsilon}^{s}(x)$.

Assume now that $s<0$ and put $s^{\prime}=-s$. Define $g \in \operatorname{Rep}[0, \infty)$ by

$$
g(t)= \begin{cases}\frac{h\left(s^{\prime}\right)+s^{\prime}}{s^{\prime}} t & \text { for } 0 \leq t \leq s^{\prime} \\ h(t)+s^{\prime} & \text { for } s^{\prime} \leq t\end{cases}
$$

For $0 \leq t \leq s^{\prime}$ we have

$$
\begin{aligned}
d(z g(t), x t) \leq & d(z g(t), z h(t))+d(z h(t), z(h(t)-s)) \\
& +d(y h(t), x t) \leq \varepsilon / 3+\varepsilon / 3+\varepsilon^{\prime} \leq \varepsilon,
\end{aligned}
$$

where the second term is $\leq \varepsilon / 3$ by (1) since $|s| \leq \tau<\tau_{1}$, the third is $\leq \varepsilon^{\prime}$ by (3) and the first is $\leq \varepsilon / 3$ since from (2) we have $h(t) \leq h(\tau) \leq 2 \tau<\tau_{1}$ and $g(t) \leq h\left(s^{\prime}\right)+s^{\prime} \leq h(\tau)+\tau \leq 3 \tau<\tau_{1}$. For $s^{\prime} \leq t$ we have, by $(3), d(z g(t), x t)=d(y h(t), x t) \leq \varepsilon^{\prime}<\varepsilon$. Hence $z \in W_{\varepsilon}^{s}(x)$. This proves Lemma 3.1.

Note that Lemma 3.1 holds for any flow without fixed points on a compact metric space.

3.2. Proposition. The set of sinks (sources) is open.

Proof. We apply Theorem 2.3. Fix $r>0$ and let $0<2 \varepsilon \leq \varepsilon_{r}$. Let $x$ be a sink. Then $x \in \operatorname{int} W_{\varepsilon}^{s}(x)$. Any $y \in \operatorname{int} W_{\varepsilon}^{s}(x)$ is also a sink since by the triangle inequality $W_{\varepsilon}^{s}(x) \subset W_{2 \varepsilon}^{s}(y)$ and thus int $W_{2 \varepsilon}^{s}(y) \neq \emptyset$. This proves the proposition. 
In the proofs of Propositions 3.4 and 3.5 we use the concept of a local cross-section and the concept of coordinates. We shall show a certain kind of compatibility of these concepts.

$S \subset X$ is a local cross-section of a time $\xi>0$ for the flow $F$ if $S$ is closed and $S \cap x[-\xi, \xi]=\{x\}$ for all $x \in S$. For such an $S, F$ maps $S \times[-\xi, \xi]$ homeomorphically onto the compact set $S[-\xi, \xi]$. Let $S^{*}=S \cap \operatorname{int} S[-\xi, \xi]$. Then $S^{*}(-\varepsilon, \varepsilon)$ is open for any $\varepsilon>0$. By a theorem of Whitney ([13], p. 270) for each $x \in X$ there exists a local cross-section $S$ of a time $\xi>0$ such that $x \in S^{*}$.

Assume that $S$ is a local cross-section of a time $\xi>0$. Let $\pi: S[-\xi, \xi] \rightarrow$ $S$ and $\tau: S[-\xi, \xi] \rightarrow[-\xi, \xi]$ be continuous maps defined by

$$
\left(\left.F\right|_{S \times[-\xi, \xi]}\right)^{-1}(x)=(\pi(x), \tau(x)) .
$$

Let $0<r<\xi$ and $\varepsilon>0$. Assume that $x, y \in S[-\xi, \xi]$ are such that $[x, y]_{r, \varepsilon}$ is defined and $[x, y]_{r, \varepsilon} \subset S[-\xi, \xi]$. Then $\pi\left([x, y]_{r, \varepsilon}\right)$ consists of a single point, which does not depend on $r$ and $\varepsilon$. We define

$$
\langle x, y\rangle=\pi\left([x, y]_{r, \varepsilon}\right)
$$

provided the right hand side makes sense. The properties of $\langle$,$\rangle are listed$ in the following:

3.3. Lemma. Let $a \in X$ and let $S$ be a local cross-section of a time $\xi>0$ such that $a \in S^{*}$. Let $0<r<\xi$ and $0<\varepsilon \leq \varepsilon_{0}$, where $\varepsilon_{0}$ corresponds to $r$ in the sense of Theorem 2.1. Let $r<\eta<\xi$. There exist numbers $\gamma_{0} \geq \gamma_{1} \geq \gamma_{2} \geq \gamma_{3} \geq \gamma_{4}>0$ such that:

(i) For $x, y \in B\left(a, \gamma_{0}\right),[x, y]_{r, \varepsilon}$ is defined and $[x, y]_{r, \varepsilon} \subset S(-\eta, \eta)$.

(ii) $\langle$,$\rangle is continuous on B\left(a, \gamma_{0}\right) \times B\left(a, \gamma_{0}\right)$.

(iii) For $x, y \in B\left(a, \gamma_{1}\right)$

$$
\langle x, y\rangle=W_{\varepsilon}^{s}(x) \cap W_{\varepsilon}^{u}(y) \cap S .
$$

(iv) For $x \in B_{S}\left(a, \gamma_{2}\right)$

$$
\begin{aligned}
& W_{\varepsilon}^{s}(x) \cap B_{S}\left(x, \gamma_{2}\right)=\left\{y \in B_{S}\left(x, \gamma_{2}\right): y=\langle x, y\rangle\right\}, \\
& W_{\varepsilon}^{u}(x) \cap B_{S}\left(x, \gamma_{2}\right)=\left\{y \in B_{S}\left(x, \gamma_{2}\right): y=\langle y, x\rangle\right\},
\end{aligned}
$$

where $B_{S}(a, \lambda)=B(a, \lambda) \cap S$.

(v) For $x, y, z, w \in B\left(a, \gamma_{3}\right)$

$$
\begin{gathered}
\langle x, x\rangle=x, \quad\langle\langle x, y\rangle, z\rangle=\langle x, z\rangle=\langle x,\langle y, z\rangle\rangle, \\
\langle\langle x, y\rangle,\langle z, w\rangle\rangle=\langle x, w\rangle
\end{gathered}
$$

and all the above symbols are well-defined.

(vi) There exists a neighborhood $U$ of a in $S$ such that the map

$$
\mathcal{X}_{a}: W_{\varepsilon}^{s}(a) \cap B_{S}\left(a, \gamma_{4}\right) \times W_{\varepsilon}^{u}(a) \cap B_{S}\left(a, \gamma_{4}\right) \rightarrow U
$$

defined by $\mathcal{X}_{a}(x, y)=\langle y, x\rangle$ is a homeomorphism. 
Proof. (i) Let $x, y \in B(a, \delta / 2)$, where $\delta$ corresponds to $\varepsilon$ in the sense of Theorem 2.1. Then $[x, y]_{r, \varepsilon}$ is defined. As $a \in S^{*}$, we have $a t \in \operatorname{int} S(-\eta, \eta)$ for every $|t| \leq r$, so by compactness of the interval $[-r, r]$ there exists $\beta>0$ such that $\bigcup_{|t| \leq r} B(a t, \beta) \subset S_{(-\eta, \eta)}$. Let $0<\gamma_{0}<\delta / 2$ correspond to this $\beta$ according to Theorem 2.1(c). For $x, y \in B\left(a, \gamma_{0}\right)$ we have $[x, y]_{r, \varepsilon} \times[a, a]_{r, \varepsilon} \subset$ $D_{\beta, r}$ and in particular $[x, y]_{r, \varepsilon} \subset S(-\eta, \eta)$.

(ii) This follows from (i), (4) and the continuity condition (Theorem 2.1(c)).

(iii) Let $\varepsilon^{\prime}>0$ and $\tau>0$ correspond to $\varepsilon$ as in Lemma 3.1. Assume $\varepsilon^{\prime}<\varepsilon, \tau<\xi$. Condition (i) that we have just proved provides $\gamma_{1} \leq \gamma_{0}$ such that for all $x, y \in B\left(a, \gamma_{1}\right)$ we have $[x, y]_{\tau / 2, \varepsilon^{\prime}} \subset S(-\tau, \tau)$. Let $z \in$ $[x, y]_{\tau / 2, \varepsilon^{\prime}}$. There exists $|t| \leq \tau$ such that $z t \in S$. So $\langle x, y\rangle(-t)=z \in$ $W_{\varepsilon^{\prime}}^{s}(x) \cap W_{\varepsilon^{\prime}}^{u}(y)$, hence by the choice of $\varepsilon^{\prime}$ and $\tau,\langle x, y\rangle \in W_{\varepsilon}^{s}(x) \cap W_{\varepsilon}^{u}(y)$. As $W_{\varepsilon}^{s}(x) \cap W_{\varepsilon}^{u}(y) \cap S$ contains at most one point we have proved the desired condition.

(iv) This easily follows from (iii) if we put $\gamma_{2}=\gamma_{1} / 2$.

(v) The first equality is obvious. This together with condition (ii) implies that for $x, y, z, w$ sufficiently close to $a,\langle x, y\rangle,\langle y, z\rangle$ and $\langle z, w\rangle$ are arbitrarily close to $a$. So $\langle\langle x, y\rangle, z\rangle,\langle x,\langle y, z\rangle\rangle$ and $\langle\langle x, y\rangle,\langle z, w\rangle\rangle$ are defined if $x, y, z, w$ are close to $a$. We can choose $\gamma_{1}^{\prime}>0$ for $\varepsilon / 2$ so that $\langle u, v\rangle=W_{\varepsilon / 2}^{s}(u) \cap W_{\varepsilon / 2}^{u}(v) \cap S$ for $u, v \in B\left(a, \gamma_{1}^{\prime}\right)$. Let $0<\gamma_{3}<\min \left(\gamma_{0}, \gamma_{1}\right)$ be such that $\langle x, y\rangle \in B\left(a, \gamma_{1}^{\prime}\right)$ if $x, y \in B\left(a, \gamma_{3}\right)$. Let $u=\langle\langle x, y\rangle, z\rangle$. Then $u \in W_{\varepsilon / 2}^{s}(\langle x, y\rangle)$ and since $\langle x, y\rangle \in W_{\varepsilon / 2}^{s}(x)$ we have $u \in W_{\varepsilon}^{s}(x)$. Also $u \in W_{\varepsilon / 2}^{u}(z) \subset W_{\varepsilon}^{u}(z)$, hence $u=\langle x, z\rangle$. We may decrease $\gamma_{3}$ if necessary to get the remaining equalities.

(vi) Pick $0<\gamma_{4} \leq \min \left(\gamma_{2}, \gamma_{3}\right)$ and let

$$
U=\mathcal{X}_{a}\left(W_{\varepsilon}^{s}(a) \cap B_{S}\left(a, \gamma_{4}\right) \times W_{\varepsilon}^{u}(a) \cap B_{S}\left(a, \gamma_{4}\right)\right) .
$$

We show that $\mathcal{X}_{a}$ is injective and hence bijective onto its image. For let $\langle y, x\rangle=\langle\bar{y}, \bar{x}\rangle$, where $x, \bar{x} \in W_{\varepsilon}^{s}(a) \cap B_{S}\left(a, \gamma_{4}\right), y, \bar{y} \in W_{\varepsilon}^{u}(a) \cap B_{S}\left(a, \gamma_{4}\right)$. By (v) we have $\langle\langle y, x\rangle, a\rangle=\langle y, a\rangle$ and $\langle\langle\bar{y}, \bar{x}\rangle, a\rangle=\langle\bar{y}, a\rangle$. Hence by (iv), $\langle y, a\rangle=y$ and $\langle\bar{y}, a\rangle=\bar{y}$, which means $y=\bar{y}$. Similarly $x=\bar{x}$. It is enough to show that $U$ is a neighborhood of $a$ in $S$. Let $\delta>0$ be such that $d(a,\langle z, a\rangle) \leq \gamma_{4}$ and $d(a,\langle a, z\rangle) \leq \gamma_{4}$ if $d(a, z) \leq \delta$. Let $z \in B_{S}(a, \delta)$. Put $x=\langle a, z\rangle, y=\langle z, a\rangle$. (v) implies that $x=\langle a, z\rangle=\langle a,\langle a, z\rangle\rangle=\langle a, x\rangle$ and similarly $y=\langle y, a\rangle$. Now, by (iv), $x \in W_{\varepsilon}^{s}(a) \cap B_{S}\left(a, \gamma_{4}\right)$ and $y \in$ $W_{\varepsilon}^{u}(a) \cap B_{S}\left(a, \gamma_{4}\right)$. Again by $(\mathrm{v}), \mathcal{X}_{a}(x, y)=\langle\langle z, a\rangle,\langle a, z\rangle\rangle=z$. Hence $B_{S}(a, \delta) \subset U$ and the proof is complete.

3.4. Proposition. If $X$ is locally connected then the set of sinks (sources) is closed.

Pr o of. Let $a$ belong to the closure of the set of sinks. Assume that it is 
not a sink. Then for a fixed $r>0,0<\varepsilon \leq \varepsilon_{r}$ and a neighborhood $H$ of $a$, $W_{\varepsilon}^{u}(a) \cap H \backslash x[-r, r] \neq \emptyset$. Let $S$ be a local cross-section of a time $\xi>0$ such that $a \in S^{*}$. Let $0<r<\xi / 2,0<\varepsilon \leq \varepsilon_{r}$ and pick $\varepsilon^{\prime}<\varepsilon, \tau<r$ corresponding to this $\varepsilon$ according to Lemma 3.1. We apply Lemma 3.3 with $\varepsilon^{\prime}$ instead of $\varepsilon$. Condition (vi) provides an appropriate neighborhood $U$ of $a$ in $S$. Let $W$ be a connected neighborhood of $a$ such that $W \subset U(-\tau, \tau)$. Let $p_{s}$ and $p_{u}$ denote the continuous projections from $U$ onto $W_{\varepsilon^{\prime}}^{s}(a) \cap B_{S}\left(a, \gamma_{4}\right)$ and $W_{\varepsilon^{\prime}}^{u}(a) \cap B_{S}\left(a, \gamma_{4}\right)$, respectively, i.e. $\mathcal{X}_{a}\left(p_{s}(z), p_{u}(z)\right)=z$ for $z \in U$. The set $V=p_{u}(\pi(W))$ is a connected neighborhood of $a$ in $W_{\varepsilon^{\prime}}^{u}(a) \cap B_{S}\left(a, \gamma_{4}\right)$ and as $a$ is not a sink, any point of $V$ is non-isolated in $W_{\varepsilon^{\prime}}^{u}(a) \cap B_{S}\left(a, \gamma_{4}\right)$. Let $b \in W$ be a sink and let $\pi(b)=\langle y, x\rangle$ with $y \in V, x \in W_{\varepsilon^{\prime}}^{s}(a) \cap B_{S}\left(a, \gamma_{4}\right)$. There exists a sequence $y_{n} \rightarrow y, n \rightarrow \infty, y \neq y_{n} \in V$. Let $b_{n}=z_{n}(-\tau(b))$, where $z_{n}=\left\langle y_{n}, x\right\rangle$. We see that $\left\langle z_{n}, \pi(b)\right\rangle=\left\langle\left\langle y_{n}, x\right\rangle,\langle y, x\rangle\right\rangle=\left\langle\left\langle y_{n}, x\right\rangle, x\right\rangle=$ $\left\langle y_{n}, x\right\rangle=z_{n}$, hence $z_{n} \in W_{\varepsilon^{\prime}}^{u}(\pi(b))$, so by the choice of $\varepsilon^{\prime}$ and $\tau, b_{n} \in W_{\varepsilon}^{u}(b)$. Continuity of $\langle$,$\rangle and of the flow implies that b_{n} \rightarrow b$ as $n \rightarrow \infty$. On the other hand, $y_{n} \neq y$ implies $z_{n} \neq \pi(b)$. Hence $b_{n} \notin b[-r, r]$ since $r<\xi / 2$ and $|\tau(b)|<r$. This yields a contradiction since by Theorem $2.3(\mathrm{c}), b$ is not a sink.

3.5. Proposition. If $X$ is connected, locally connected and is not a single orbit, then any point in $X$ is flow non-isolated.

Proof. Assume that $x \in X$ is flow isolated. So there exist a neighborhood $U$ of $x$ and $r>0$ with $U \subset x[-r, r]$. This implies that any point on the orbit $x \mathbb{R}$ is flow isolated and $x \mathbb{R}=\bigcup_{t \in \mathbb{R}} U t$ is an open set. Since $X$ is connected and contains more than one orbit, $x \mathbb{R}$ cannot be closed. Let $y$ be a limit point of $x \mathbb{R}, y \notin x \mathbb{R}$. Let $S$ be a local cross-section of a time $\xi>0$ with $x \in S^{*}$. Let $W$ be a connected neighborhood of $x$ with $W \subset S^{*}(-\xi, \xi)$. So $\pi(W)$ is connected in $S$. To this $W$ we choose $t_{0} \in \mathbb{R}$ with $x t_{0} \in W$. Then $\pi\left(x t_{0}\right) \in \pi(W)$. Now $x t_{0} \neq y$ and $x t_{0}$ is isolated in $S^{*}$, contrary to the connectedness of $\pi(W)$.

Proof of Theorem 1.1. Proposition 3.5 implies that any point is flow non-isolated and hence the sets of saddles, sources and sinks are pairwise disjoint. This, by Propositions 3.2 and 3.4, means that the proof will be complete if we show that the set of sinks (sources) is not the whole space $X$.

Assume, then, that any point of $X$ is a sink. Fix $x \in X$. As $x$ is a sink its $\omega$-limit set forms a periodic orbit $K$ and any point in $K$ is a sink. Proposition 2.6 implies that $K$ is a basic set. By Theorem 2.4, $K$ is open in $\Omega$ and, being a periodic orbit, it is closed in $X$. Therefore $\Omega$ is not equal to $X$. Let $0<\varepsilon \leq \varepsilon_{1}$ with $\varepsilon_{1}$ given in Theorem 2.5. For any $y \in X$, $y t \rightarrow \Omega$ as $t \rightarrow-\infty$, so $X=\bigcup_{i=1}^{r} \bigcup_{n \in \mathbb{N}} W_{\varepsilon}^{u}\left(\Omega_{i}\right) n$, by Theorem 2.4. Since each $W_{\varepsilon}^{u}\left(\Omega_{i}\right)$ and hence $W_{\varepsilon}^{u}\left(\Omega_{i}\right) n$ is closed, by the Baire Category Theorem $\operatorname{int} W_{\varepsilon}^{u}\left(\Omega_{i_{0}}\right) \neq \emptyset$ for some basic set $\Omega_{i_{0}}$. By Theorem 2.5 applied to the 
reverse flow, $W^{s}\left(\Omega_{i_{0}}\right)=\Omega_{i_{0}}$. Let $y \in \Omega_{i_{0}}$. Since $y$ is a sink, $\emptyset \neq \operatorname{int} W_{\varepsilon}^{s}(y) \subset$ $W_{\varepsilon}^{s}\left(\Omega_{i_{0}}\right)$ by Theorem 2.3(c), so $W^{s}\left(\Omega_{i_{0}}\right)$ is open by Theorem 2.5 . Therefore $\Omega_{i_{0}}$ is open and since it is closed as a basic set, it must be equal to $X$. But we have just proved that $\Omega_{i_{0}} \subset \Omega \neq X$. This contradiction completes the proof.

4. Coordinates. Most of the proof of our main result (Theorem 1.1) as well as a large part of paper [6] depend on the coordinates that have been identified in Theorem 2.1 (Proposition 2.9 in [6]). In this section we study this concept in more detail.

4.1. Definition. The flow $F$ has coordinates if for any $r>0$ there exists $\varepsilon_{0}>0$ with the property that for every $0<\varepsilon \leq \varepsilon_{0}$ there exist $\delta>0$ and a map $[,]_{r, \varepsilon}: B_{\delta} \rightarrow 2^{X}$ such that:

(a) for any $(x, y) \in B_{\delta}$

$$
[x, y]_{r, \varepsilon}=W_{\varepsilon}^{s}(x) \cap W_{\varepsilon}^{u}(y) \neq \emptyset,
$$

(b) for any $(x, y) \in B_{\delta}$ and $z, w \in[x, y]_{r, \varepsilon}$ there exists $|t| \leq r$ with $w=z t$.

The above definition differs from the definition of canonical or hyperbolic coordinates known for Axiom A flows. Hyperbolic coordinates imply expansiveness [1] and the pseudo orbits tracing property [3], hence they imply coordinates. We do not, however, know whether coordinates imply hyperbolic coordinates like in the case of homeomorphisms ([7], [4]). What we want to show here is that coordinates are a topological (orientation-preserving) conjugacy invariant and are compatible via the suspension operation with coordinates defined for homeomorphisms. There is no evidence that the above two statements hold true for hyperbolic coordinates. By the way, it is easy to see that Definition 4.1 does not depend on the distance used, unlike the definition of hyperbolic coordinates.

Recall that flows $F$ and $G$ are conjugate with preserved orientation if there is a homeomorphism $\lambda$ which maps orbits of $F$ onto orbits of $G$ and which preserves orientation of the orbits.

4.2. Proposition. Assume that flows $F$ and $G$ are conjugate with preserved orientation. Then $F$ has coordinates if and only if $G$ does.

4.3. Lemma. If a flow has coordinates then it is expansive.

Proof. This follows from Definition 4.1(b) if for $r>0$ we define $e=\min \left(\varepsilon_{0}, \delta\right)$ where $\varepsilon_{0}$ corresponds to $r$ and $\delta$ to $\varepsilon_{0}$ in the sense of that definition.

4.4. Lemma (Corollary 4 in [2]). Expansiveness is a conjugacy invariant. 
4.5. LemmA. Let $F$ and $G$ be conjugate with preserved orientation. Then:

(a) $G$ has no fixed points.

(b) For any $x \in X$ there is $\alpha_{x} \in \operatorname{Rep}(\mathbb{R})$ such that $G\left(\lambda(x), \alpha_{x}(t)\right)=$ $\lambda(F(x, t))$ for all $t \in \mathbb{R}$.

Proof. This is done, in fact, in the proof of Corollary 4 in [2].

Proof of Theorem 4.2. Assume, for instance, that $F$ has coordinates. By Lemmas 4.3 and 4.4, $G$ is expansive. Fix $r>0$ and let $\varepsilon_{0}^{\prime}=e^{\prime} / 2$ where $e^{\prime}$ is chosen for $r$ by expansiveness of $G$. Let $0<\varepsilon^{\prime} \leq \varepsilon_{0}^{\prime}$. Let $\varepsilon_{0}>0$ be chosen for $r$ according to Definition 4.1 and pick $\varepsilon$ with $0<\varepsilon \leq \varepsilon_{0}$ such that $d(x, y) \leq \varepsilon$ implies $d(\lambda(x), \lambda(y)) \leq \varepsilon^{\prime}$. For this $\varepsilon$ pick $\delta>0$ according to Definition 4.1 and $\delta^{\prime}>0$ such that $d(u, v) \leq \delta^{\prime}$ implies $d\left(\lambda^{-1}(u), \lambda^{-1}(v)\right) \leq \delta$. Let $(u, v) \in B_{\delta^{\prime}}$. Lemma 4.5(b) easily implies that $W_{\varepsilon^{\prime}}^{s}(u, G) \cap W_{\varepsilon^{\prime}}^{u}(v, G) \neq \emptyset$ for $(u, v) \in B_{\delta^{\prime}}$, so condition (a) in Definition 4.1 is satisfied by the flow $G$, and condition (b) in this definition easily follows from expansiveness and the choice of $\varepsilon_{0}^{\prime}$.

Let $f: Y \rightarrow Y$ be a homeomorphism of a compact metric space $(Y, p)$. We say that $f$ has coordinates if there exists $\mu_{0}>0$ such that for any $0<\mu \leq$ $\mu_{0}$ there exists $\lambda>0$ such that for any $(x, y) \in B_{\lambda}, W_{\mu}^{s}(x, f) \cap W_{\mu}^{u}(y, f)$ is a single point (see [5]). Here, $W_{\mu}^{s}(x, f)=\left\{y \in Y: p\left(f^{n} x, f^{n} y\right) \leq \mu\right.$ for $\left.n \geq 0\right\}$ and $W_{\mu}^{u}(x, f)=W_{\mu}^{s}\left(x, f^{-1}\right)$. It is known that $f$ has coordinates if and only if $f$ has hyperbolic coordinates and if and only if $f$ is expansive and has the pseudo orbits tracing property $([4],[5])$.

We briefly recall the construction of the suspension flow (see [2] or [9] for more details). Let $f: Y \rightarrow Y$ be a homeomorphism of a compact metric space $(Y, p)$. Let $X=(Y \times[0,1]) / \sim$, where the equivalence relation $\sim$ identifies $(y, 1)$ with $(f y, 0)$. Define a flow $F: X \times \mathbb{R} \rightarrow X$ by $F((x, t), s)=$ $\left(f^{n} x, r\right)$, where $n=[t+s], r=t+s-[t+s]$. One can show that $X$ is compact and the homeomorphism $f$ is topologically conjugate to the timeone map of the flow $F$, i.e. the map $X \ni x \rightarrow F(x, 1) \in X$. More specific information is contained in the following:

4.6. Lemma. There exists a distance $d$ on $X$ compatible with the topology of $X$ such that the following conditions hold:

(a) for $x, y \in Y, d((x, 0),(y, 0))=\min (p(x, y), p(f x, f y))$,

(b) for $(x, s),(y, t) \in X$ and $0<\varepsilon<1 / 4$, if $d((x, s),(y, s)) \leq \varepsilon$, then either $|s-t| \leq \varepsilon$ or $|1+s-t| \leq \varepsilon$ or $|1+t-s| \leq \varepsilon$,

(c) for each $\alpha>0$ there exists $\beta>0$ such that $d((x, 1 / 2),(y, t)) \leq \beta$ implies $p(x, y) \leq \alpha$.

Proof. See [2] or [9] for the construction of the metric $d$. Condition (a) easily follows from this construction. Condition (b) is established in 
Lemma 2.4 of [9], and (c) is a simple consequence of Lemma 2.5 in [9].

4.7. Proposition. Let $f$ be a homeomorphism of a compact metric space $Y$ and $F$ its suspension flow. Then $f$ has coordinates if and only if $F$ does.

Proof. Assume that $f$ has coordinates. By Theorem 4.3 in [5], $f$ is expansive and has the pseudo orbits tracing property. Then $F$ is expansive (Theorem 6 in [2]) and has the pseudo orbits tracing property (Theorem 2 in [9]). By Proposition 2.9 in [6] (Theorem 2.1 in the present paper) $F$ has coordinates.

Conversely, assume that $F$ has coordinates. By Lemma 4.3 it is expansive, so by Theorem 6 in [2], $f$ is expansive, i.e. there exists $e>0$ such that $p\left(f^{n} x, f^{n} y\right) \leq e$ for all $n \in \mathbb{Z}$ implies $x=y$. Let $\mu_{0}=\min \left(e / 2, \varepsilon_{0}\right)$, where $\varepsilon_{0}$ corresponds to $r=1 / 4$ in the sense of Definition 4.1. Let $0<\mu \leq \mu_{0}$. We pick $\varepsilon<\min (\mu, 1 / 4)$ such that $d((x, 1 / 2),(y, t)) \leq \varepsilon$ implies $p(x, y) \leq \mu$ (Lemma 4.6(c)). Let $\delta>0$ correspond to this $\varepsilon$ according to Definition 4.1. Pick $\lambda>0$ such that $p(x, y) \leq \lambda$ implies $d((x, 1 / 2),(y, 1 / 2)) \leq \delta$ (by Lemma 4.6(a) and continuity of $F$ ).

Let $(x, y) \in B_{\lambda}$. By expansiveness of $f, W_{\mu}^{s}(x, f) \cap W_{\mu}^{u}(y, f)$ contains at most one point. We prove that $W_{\mu}^{s}(x, f) \cap W_{\mu}^{u}(y, f) \neq \emptyset$. By the choice of $\lambda$ and $\delta$ we know that $W_{\varepsilon}^{s}((x, 1 / 2)) \cap W_{\varepsilon}^{u}((y, 1 / 2))$ contains at least one point $\left(z, t_{0}\right)$. We shall show that $z \in W_{\mu}^{s}(x)$; the proof that $z \in W_{\mu}^{u}(y)$ is the same. Since $\left(z, t_{0}\right) \in W_{\varepsilon}^{s}((x, 1 / 2))$ there exists $h \in \operatorname{Rep}[0, \infty)$ such that

$$
d\left(F\left(\left(z, t_{0}\right), h(t)\right), F((x, 1 / 2), t)\right) \leq \varepsilon \quad \text { for } t \geq 0 .
$$

Letting $t=0$ we have $d\left(\left(z, t_{0}\right),(x, 1 / 2)\right) \leq \varepsilon<1 / 4$, which implies $\mid t_{0}-$ $1 / 2 \mid<1 / 4$ by Lemma $4.6(\mathrm{~b})$ and $p(z, x) \leq \mu$ by Lemma $4.6(\mathrm{c})$. Then we also have

$$
d\left(F\left(\left(z, t_{0}\right), h(1)\right), F((x, 1 / 2), 1)\right) \leq \varepsilon<1 / 4 .
$$

Clearly, $F((x, 1 / 2), 1)=(f x, 1 / 2)$ and $F\left(\left(z, t_{0}\right), h(1)\right)=\left(f^{n} z, t_{1}\right)$, with $n=$ $\left[t_{0}+h(1)\right], t_{1}=t_{0}+h(1)-\left[t_{0}+h(1)\right]$. Lemma 4.6(b) implies $\left|t_{1}-1 / 2\right|<1 / 4$. Moreover, as $h$ increases and is continuous, Lemma 4.6(a) and (5) guarantee that $n=1$ and this means

$$
d\left(\left(f z, t_{1}\right),(f x, 1 / 2)\right) \leq \varepsilon \quad \text { with }\left|t_{1}-1 / 2\right|<1 / 4,
$$

so we have $p(f z, f x) \leq \mu$. Now, we may repeat the above argument to get $p\left(f^{2} z, f^{2} x\right) \leq \mu$ and so on, which proves that $z \in W_{\mu}^{s}(x, f)$. The proof is complete.

\section{References}

[1] R. Bowen, Periodic orbits for hyperbolic flows, Amer. J. Math. 94 (1972), 1-37. 
[2] R. Bowen and P. Walters, Expansive one-parameter flows, J. Differential Equations 12 (1972), 180-193.

[3] J. Franke and J. Selgrade, Hyperbolicity and chain recurrence, ibid. 26 (1977), $27-36$.

[4] J. Ombach, Equivalent conditions for hyperbolic coordinates, Topology Appl. 23 (1986), 87-90.

[5] - Expansive homeomorphisms with the pseudo orbits tracing property, preprint 383, Institute of Math., Polish Acad. of Sci., 1987.

[6] - Sinks, sources and saddles for expansive flows with the pseudo orbits tracing property, Ann. Polon. Math. 53 (1991), 237-252.

[7] W. Reddy, Expansive canonical coordinates are hyperbolic, Topology Appl. 15 (1983), 205-210.

[8] W. Reddy and L. Robertson, Sources, sinks and saddles for expansive homeomorphisms with canonical coordinates, Wesleyan University, preprint.

[9] R. Thomas, Stability properties of one-parameter flows, Proc. London Math. Soc. 45 (1982), 479-505.

[10] —, Topological stability: some fundamental properties, J. Differential Equations 59 (1985), 103-122.

[11] _, Entropy of expansive flows, Ergodic Theory Dynamical Systems, 7 (1987), 611625.

[12] - Canonical coordinates and the pseudo orbit tracing property, J. Differential Equations 90 (1991), 316-343.

[13] H. Whitney, Regular families of curves, Ann. of Math. 34 (1933), 244-270.

INSTITUTE OF MATHEMATICS

JAGIELLONIAN UNIVERSITY

REYMONTA 4

30-059 KRAKÓW, POLAND 